\title{
Review on the molecular study of the Diplozoidae: analyses of currently available genetic data, what it tells us, and where to go from here
}

\author{
Quinton Marco Dos Santos and Annemariè Avenant-Oldewage*
}

\begin{abstract}
The use of molecular tools in the study of parasite taxonomy and systematics have become a substantial and crucial component of parasitology. Having genetic characterisation at the disposal of researchers has produced mostly useful, and arguably more objective conclusions. However, there are several groups for which limited genetic information is available and, coupled with the lack of standardised protocols, renders molecular study of these groups challenging. The Diplozoidae are fascinating and unique monogeneans parasitizing mainly freshwater cyprinid fishes in Europe, Asia and Africa. This group was studied from a molecular aspect since the turn of the century and as such, limitations and variability concerning the use of these techniques have not been clearly defined. In this review, all literature and molecular information, primarily from online databases such as GenBank, were compiled and scrupulously analysed for the Diplozoidae. This was done to review the information, detect possible pitfalls, and provide a "checkpoint" for future molecular studies of the family. Hindrances detected are the availability of sequence data for only a limited number of species, frequently limited to a single sequence per species, and the heavy reliance on one non-coding ribosomal marker (ITS2 rDNA) which is difficult to align objectively and displays massive divergences between taxa. Challenging species identification and limited understanding of diplozoid species diversity and plasticity are also likely restricting factors, all of which hamper the accurate taxonomic and phylogenetic study of this group. Thus, a more integrated taxonomic approach through the inclusion of additional markers, application of more rigorous morphological assessment, more structured barcoding techniques, alongside thorough capturing of species descriptions including genetypes, genophore vouchers and reference collections in open sources are encouraged. The pitfalls highlighted are not singular to the Diplozoidae, and the study of other groups may benefit from the points raised here as well.
\end{abstract}

Keywords: Monogenea, Diplozoon, Paradiplozoon, Eudiplozoon, Sindiplozoon, Inustiatus, Afrodiplozoon, Molecular taxonomy, Genetic characterisation, Fish parasites

\section{Background}

The Diplozoidae Palombi, 1949 is a fascinating and unique aquatic parasite family, with hermaphroditic monogenean pairs fusing to mature sexually, forming

${ }^{*}$ Correspondence: aoldewage@uj.ac.za

Department of Zoology, University of Johannesburg, Auckland Park, P.O.

Box 524, Johannesburg 2006, South Africa
"X"-shaped, "Siamese" organisms, allowing for crossfertilization [1] and supporting genetic diversity. They occur on the gills of their fish hosts, in the interhemibrachial septum of the primary lamellae, where they feed on blood and tissue, attached with specialised organs situated at their posterior ends called haptors. Within the Diplozoidae, haptors are usually disc-shaped structures with clamps, hooks, or cup-like structures, the number 
and shape of which is subfamily- and genus-specific. The hardened internal structures, or sclerites, of the hooks and clamps are historically considered the most crucial morphological characteristics for species identification and differentiation [2-4]. Unlike other monogeneans, species of the Diplozoidae lack sclerotised genitalia and only possess sclerites in their haptors. These sclerites are small and often difficult to accurately visualise due to their orientation and position within tissue. This, alongside the lack of other robust taxonomic features, hinder accurate identification and delimitation of species in this family. With the exception of selected species, diplozoids are considered to be host specific. They occur mostly on cyprinid fishes in freshwater systems of Eurasia and Africa, although in Africa they also parasitize characid species [5-8]. A few accounts of diplozoids infecting other fish families do exist, like Acipenseridae [3], Anguillidae [9, 10], Cichlidae [11, 12], Cottidae [3, 13], Esocidae [3], Gasterosteidae [3, 14], Gobiidae [3], Lotidae $[3,13]$, and Percidae $[3,9,10,15]$, even from brackish $[9,10]$ or marine environments [14]. Unfortunately, the majority of these records are vague, for unidentified diplozoid taxa, or have not been noted again. However, these records are intriguing and would benefit from further investigation, especially those from the Anguillidae and Percidae $[3,9,10,15]$.

Molecular approaches to the study of diplozoid parasites is a relatively new field, with members of the family only included in phylogenetic studies since 2000 [16]. Thus, only a handful of studies concerning the genetic makeup of diplozoids have been produced and 143 sequences are currently available in public data repositories and published literature (Additional file 1: Table S1). However, these sequences are representative of only 32 diplozoid taxa (as well as five unidentified species) which is less than a third of known species. The current number of known diplozoid species is uncertain as roughly 100 species have been described, of which 17 have been synonymised, 6 are considered species inquirenda, 20 have not been confirmed, and 55 are generally accepted, with several unidentified species mentioned in literature. Of the two subfamilies in the Diplozoidae, all genera of the Diplozoinae (Diplozoon von Nordmann, 1832; Paradiplozoon Akhmerov, 1974; Sindiplozoon Khotenovsky, 1981; Inustiatus Khotenovsky, 1978; Eudiplozoon Khotenovsky, 1985) have representative sequence data, constituting the majority of taxa for which data are available (31 species, 142 sequences). A single sequence for a member of the second subfamily, Neodiplozoinae, has recently been included (Afrodiplozoon polycotyleus (Paperna, 1973)) [17]. Thus, the only diplozoid genus for which no published molecular information is available is the monotypic Neodiplozoon Tripathi, 1960 (Neodiplozoon barbi
(Tripathi, 1957)) which is the second genus within the Neodiplozoinae. Most sequence data (116 sequences) are for the second internal transcribed spacer region (ITS2) between the $5.8 \mathrm{~S}$ and $28 \mathrm{~S}$ (large) nuclear ribosomal subunits. Seven of the published ITS2 sequences include a short portion of the $28 \mathrm{~S}$, while five sequences include a short portion of $18 \mathrm{~S}$ and the full ITS1 and $5.8 \mathrm{~S}$ regions, alongside the ITS2 rDNA region. The remaining 27 sequences are derived from eight gene regions: ten sequences for the universal barcoding region of the mitochondrial cytochrome $c$ oxidase subunit 1 ( $\operatorname{cox} 1)$ gene, five sequences for the large $28 \mathrm{~S}$ ribosomal gene, three sequences for the small $18 S$ ribosomal gene, three sequences for cysteine peptidase regions (L1, L3 and B), three mitogenome sequences (from which $\operatorname{cox} 1$ can be mined) and single sequences for a cysteine peptidase inhibitor (stefin), a Kunitz-type protease inhibitor (serine), and a serine protease inhibitor (serpin) respectively.

The first molecular investigations incorporating members of the Diplozoidae [16, 18-20] were submitted prior to the proposal for a single DNA fragment for a DNA barcoding framework [21], and mostly aimed at assessing the evolutionary relationships among monogenean groups and in relation to other Platyhelminthes. The large subunit (28S) rRNA gene was used in most of these studies, with Jovelin \& Justine [18] also amplifying the cox 1 gene and Littlewood \& Olson [19] including $18 S$ rRNA gene. Using $28 S$ rDNA, both Jovelin \& Justine [18] and Olson \& Littlewood [20] found diplozoid taxa to group within the polyopisthocotylean monogeneans, in the Oligochoinea (non-polystomatid polyopisthocotyleans). In both cases, the Discocotylinea, which includes the Diplozoidae, showed a close relationship to the Microcotylinea. Littlewood \& Olson [19] do not refer to the position of diplozoids specifically as they only studied the relationships of the greater Polyopisthocotylea in relation to other Platyhelminthes using $18 \mathrm{~S}$ rDNA. Jovelin \& Justine [18] noted the lack of resolution when attempting to infer evolutionary relationships between distantly related monogenean taxa using the $\operatorname{cox} 1$ gene due to the saturation of the cox 1 alignment. Each of the above-mentioned studies produced a single sequence per marker. The sequences, five in total, represented two diplozoid species. The cox 1 , $28 S$ and $18 S$ sequences produced by Jovelin \& Justine [18], Littlewood \& Olson [19] and Olson \& Littlewood [20] were for Eudiplozoon kamegaii Nishihira \& Urabe, 2020 (recently separated from Eudiplozoon nipponicum (Goto, 1891) [22]), while the $28 S$ sequence produced by Mollaret et al. [16] was only identified as a Diplozoon sp. Additionally, the $28 S$ sequences deposited varied in length and coverage. Recently, sequences for several coding regions of mitochondrial DNA have been published. Jedličková et al. [23, 24] produced three sequences for 
endopeptidases for E. kamegaii. These enzymes (cathepsin L (L1 and L3) and B) produced phylogenies which distantly related this diplozoid to other Platyhelminthes and the authors hinted at some interesting clues about the evolution of these parasites. Sequence data for three protease (peptidase) inhibitors have also been produced for the same species (E. kamegaii). These include the cysteine peptidase inhibitor (stefin) analysed by Ilgová et al. [25], the Kunitz protease inhibitor (KT1) studied by Jedličková et al. [26], and the Serine protease inhibitor (serpin) studied by Roudnický et al. [27]. As most of the abovementioned sequence data were for a single species, little can be deduced regarding the barcoding potential of the relative regions. Zhang et al. [28] analysed the mitogenomes of three distinct diplozoids and constructed phylogenies based on all available mitogenomic data for Platyhelminthes, 21 species in total. In contrast to previous studies, diplozoid (Discocotylinea) taxa grouped sister to species of the Chauhaneidae (Gastrocotylinea) and Microcotylidae (Microcotylinea) in all cases, indicating that mitogenomic analyses might provide more insightful results for interrelationships of monogenean suborders [28].

As mentioned, most sequence data for the Diplozoidae were generated for the ITS2 fragment. The first of these sequences were published in 2001, when both Sicard et al. [29] and Matejusová et al. [4] used this marker to distinguish diplozoid species. These authors did not state why this marker was selected for their investigations, but both concluded that ITS2 rDNA can be applied to discriminate between distinct diplozoid species. Since then, almost all studies that included molecular assessment of diplozoids used only this marker, particularly studies related to taxonomy and phylogeny. The discriminatory power of ITS 2 rDNA has been used to identify, describe, and re-describe several diplozoid species. It has been speculated that the identification of the parasites from which some of these ITS2 sequences were generated, as well as those for other markers, are incorrect [22, 30-32], causing confusion and controversy, and thus requiring revision. Additionally, the usefulness of other markers to study cryptic families like the Diplozoidae has been neglected and should be assessed alongside a revision of ITS2 rDNA.

Given the limited amount of data published, the taxonomic uncertainty of some published sequences, and the disparity of the selection of genetic markers to study these parasites, the time is ripe for a comprehensive revision of available molecular information on the Diplozoidae. The aim of this review was to collect, compile, analyse, and scrutinise the currently available taxonomic genetic data for the Diplozoidae. We aimed to determine if genetic studies of species of this family are on the right track, what can be gleaned from available data, how studies could be improved in the future, and what pitfalls to avoid.

\section{Barcoding for species identification}

The molecular study of the Diplozoidae for taxonomic purposes highlights two of the most pressing pitfalls of molecular taxonomy. The first is the use of different markers, or different regions of the same gene fragment, in isolated studies and for different species, producing data which are not comparable. The neglect of the universal barcoding gene, cox 1 , to study these parasites also makes it difficult to compare this family to most other organisms for which this marker has become the benchmark. Secondly, the incorrect, incomplete, and often subjective identification of the material of the published sequence data, with little or no supporting morphological data can cause much confusion. To assess the sequences and markers available, relative sequences were aligned using MEGA 7 [33] and pairwise distances (uncorrected p-distance) calculated (1000 bootstrap replicated). All sequence data for protein-coding markers are for the same species, E. kamegaii, and thus the taxonomic usefulness of these markers could not be assessed.

\section{$18 \mathrm{~S}$ rDNA}

Only three sequences containing substantial fragments of $18 \mathrm{~S}$ rDNA are available for diplozoids. The alignment of these sequences consisted of $1962 \mathrm{bp}$, with 1773 conserved, 112 variable, and no parsimony informative sites, with uncorrected p-distances between 0-7.73\% (Table 1). Two of these sequences are identical and are for E. kamegaii, AJ287510 by Littlewood \& Olson [19] and MF579987 by E. Turgay (unpublished). The similarity of these two sequences, one collected from the Czech Republic [19] and the other from Turkey (according to an unpublished conference abstract by Kircalar et al. [34]), suggest that this marker might be a suitable candidate for barcoding. They are both also markedly distinct from the sequence for Paradiplozoon hemiculteri (Ling, 1973) (KY640614; 5.94-7.73\%), which is only available from GenBank and not published otherwise. The sequences available cover similar regions of $18 \mathrm{~S}$ rDNA, but are markedly different in length as MF579987 (750

Table 1 Sequence divergence (average uncorrected p-distance in \%) for diplozoid taxa based on partial 185 rDNA

\begin{tabular}{lllll}
\hline GenBank ID & Species & & 1 & 2 \\
\hline AJ287510 & Eudiplozoon kamegaii & 1 & - & \\
MF579987 & Eudiplozoon kamegaii & 2 & 0 & - \\
KY640614 & Paradiplozoon hemiculteri & 3 & 5.94 & 7.73 \\
\hline
\end{tabular}


bp) corresponds to just more than a third of AJ287510 (1960 bp) and KY640614 (1887 bp). Five other sequences (KY290757-KY290761) contain a tiny fragment of $18 S$ rDNA (37 bp), but they do not overlap meaningfully with the aforementioned three sequences. These five sequences also include the full ITS1 and 5.8S rDNA regions, but they are the only sequences to do so and are all for the same species ( $P$. hemiculteri), thus not allowing these two regions to be assessed beyond the $0-0.59 \%$ intraspecific distances calculated.

\section{5 rDNA}

The five available sequences for $28 \mathrm{SDNA}$ of diplozoids contains either two or three "marker fragments" because different domains of this ribosomal region have been sequenced by different authors and are not always comparable. Sequences by Sicard et al. [29], Ahmad et al. $[35,36]$, and an unpublished sequence by Sofi \& Ahmad (GenBank: MF460994) include a short section of the 3'-end of 28S (C1 and D1 domains) alongside the ITS2 fragments, about $310 \mathrm{bp}$ (190 bp for MF460994). These fragments do not fully overlap with any of the exclusively $28 S$ rDNA sequences, with the closest being that by Mollaret et al. [16] of which 76.64\% (269 out of 351 bp) is covered. However, similar to the sequence by Mollaret et al. [16], they do not overlap with the fragment of Jovelin \& Justine [18] at all. Nevertheless, they were included in analyses, bringing the total sequences analysed to 12 . The produced alignment consisted of 1276 bp, with 728 conserved, 254 variable, and 123 parsimony informative sites, with uncorrected $\mathrm{p}$-distances between $0-35.09 \%$ (Table 2). Due to the inconsistent $28 S$ coverage of sequences from different species and authors, erratic sequence length (351-1133 bp), and the limited number of species for which $28 S$ data are available, it is challenging to determine whether this marker would show enough resolution to study diplozoid diversity.

The three sequences for E. kamegaii are identical for the regions where they do overlap, and as they were produced by different authors and collected from different sites and systems, show that this marker has barcoding potential due to its apparent intraspecific stability over vast geographical reaches. Following this, even though sequences AF131717 and AF369759 only overlap for 269 bp, they are identical and may indicate that they are of the same taxon. This means that we may be able to designate the identity of AF131717, which is only noted as Diplozoon sp. by its authors [16], as Diplozoon paradoxum Nordmann, 1832, especially when also taking into account that the specimens were collected from the type-host of D. paradoxum (captured in Additional file 1: Table S1). If this assumption is made, we can see that the intraspecific range of the combination of $28 \mathrm{~S} \mathrm{rDNA}$ fragments used is 0 , with the lowest interspecific distance of 0.98 observed between D. paradoxum and P. bliccae, which have been noted to be closely related but distinct taxa in previous studies $[4,29,37]$. This further supports the possible use of fragments of $28 \mathrm{~S}$ rDNA for barcoding of diplozoid species. Based on this notion, sequence KU519493 likely represents a diplozoid taxon not yet previously genetically characterised using $28 \mathrm{~S} \mathrm{rDNA}$, even possibly a new diplozoid taxon as no diplozoid has been recorded from Barilius barila (Hamilton, 1822) or Barilius bendelisis (Hamilton, 1807).

Table 2 Sequence divergence (average uncorrected p-distance in \%) for diplozoid taxa based on partial 285 rDNA

\begin{tabular}{|c|c|c|c|c|c|c|c|c|c|c|c|c|c|}
\hline & GenBank ID & Species & 1 & 2 & 3 & 4 & 5 & 6 & 7 & 8 & 9 & 10 & 11 \\
\hline 1 & AF311703 & Eudiplozoon kamegaii & - & & & & & & & & & & \\
\hline 2 & AF382037 & Eudiplozoon kamegaii & 0 & - & & & & & & & & & \\
\hline 3 & AF369758 & Eudiplozoon kamegaii & na & 0 & - & & & & & & & & \\
\hline 4 & AF369761 & Paradiplozoon bliccae & na & 5.02 & 4.58 & - & & & & & & & \\
\hline 6 & AF369760 & Paradiplozoon homoion & na & 5.34 & 4.87 & 1.31 & - & & & & & & \\
\hline 5 & AF131717 & Diplozoon sp. ${ }^{a}$ & na & 4.96 & 6.42 & 1.15 & 1.89 & - & & & & & \\
\hline 7 & AF369759 & Diplozoon paradoxum & na & 6.08 & 5.5 & 0.98 & 1.62 & 0 & - & & & & \\
\hline 8 & AF973617 & Paradiplozoon aegyptense & na & 11.79 & 10.36 & 6.54 & 7.12 & 6.39 & 5.48 & - & & & \\
\hline 9 & AF973616 & Paradiplozoon kashmirense & na & 14.58 & 14.14 & 9.95 & 10.99 & 10.14 & 9.95 & 13.09 & - & & \\
\hline 10 & MF460994 & Paradiplozoon kashmirense & na & 14.58 & 14.14 & 9.95 & 10.99 & 10.14 & 9.95 & 13.09 & 0 & - & \\
\hline 11 & MN545903 & Paradiplozoon hemiculteri & 23.61 & 18.09 & 16.67 & 17.24 & 17.42 & 13.08 & 17.74 & 23.02 & 26.35 & 26.35 & - \\
\hline 12 & KU519493 & Paradiplozoon sp. & 22.24 & 18.59 & 19.65 & 21.18 & 21.39 & 15.35 & 21.84 & 27.59 & 35.09 & 35.09 & 12.38 \\
\hline
\end{tabular}

a Following the findings presented here, this species may represent Diplozoon paradoxum Nordmann, 1832

Abbreviation: na, sequences that do not overlap 


\section{cox1 mtDNA}

Although ten sequences are available for $\operatorname{cox} 1$ gene fragments of diplozoids, a further three sequences can be mined from the mitogenomes produced by Zhang et al. [28] to bring the total to thirteen. The produced alignment consisted of $456 \mathrm{bp}$, with 274 conserved, 182 variable, and 133 parsimony informative sites, displaying uncorrected pairwise distances between $0.24-25 \%$ (Table 3). Sequences are between 362 and 441 bp long, i.e. approximately a third of a cox 1 gene [38], and far less than the 658 bp fragment generally used for barcoding [21]. Most cox1 data available for diplozoids are for Eudiplozoon (7 of 13 sequences). Unfortunately, within Eudiplozoon cox 1 data there does not seem to be a clear separation of taxa. Looking at the p-distances calculated in Table 3, sequences LC517176 and LC517177 are only separated by $0.51 \%$ and were both identified as E. nipponicum collected from Carassius auratus (Linnaeus, 1758) (Carassius buergeri grandoculis Temminck \& Schlegel, or nigorobuna, according to Nishihira \& Urabe [22]). This likely indicates that these sequences represent the same taxon. However, both these sequences are more closely related to AY009163 (2.24\%) for E. kamegaii from Cyprinus carpio Linnaeus, 1758 than to the other sequence for E. nipponicum LC517173 from C. auratus (13.38-13.82\%). Additionally, sequences LC517174 and LC517175 are separated by $3.21 \%$ even though they were both identified as E. kamegaii and collected from C. carpio, with LC 517174 only separated by $0.24 \%$ from LC517173 (E. nipponicum from C. auratus). Thus, within these six Eudiplozoon cox1 sequences, two groupings are present, LC517176, LC517177 and AY009163, separated by $0.51-2.24 \%$, and LC517173-LC517175 separated by $0.24-3.65 \%$. These two groupings are separated by $13.01-14.25 \%$. This means that there are either two species present, with up to $3.65 \%$ intraspecific distances possible in the genus, or four species, with up to $0.51 \%$ intraspecific distances possible. The final Eudiplozoon cox1 sequence, MG458326, is for an unidentified species from $C$. auratus (possibly a different host than for LC517173, LC517176-LC517177 [22]), but is 9.36$15.78 \%$ from other Eudiplozoon data and may represent a third (or fifth) undescribed, cryptic Eudiplozoon taxon. Due to the uncertainty about the species identity of some of the Eudiplozoon cox1 data, calculating intraor interspecific distances are difficult, but an intrageneric distance of $0.24-15.78 \%$ was observed. All other cox 1 diplozoid sequence data are separated by more than 17\%, except for Paradiplozoon homoion (Bychowsky \& Nagibina, 1959) (KP399595) and Paradiplozoon gracile (Reichenbach-Klinke, 1961) (KP399596). Their low distance $(0.76 \%)$ may indicate that these species are not be entirely distinct, forming part of the 'Paradiplozoon homoion-complex', which will be discussed further on. As such, from the current cox1 mtDNA available for diplozoids, this marker does not seem to be ideal for species identification. Analyses of diplozoid mitogenomes by Zhang et al. [28] allowed the authors to suggest that mitogenomes might provide better resolution for phylogenetic relationships, with some faster evolving mitochondrial genes being better suited to infer evolutionary histories and population genetics compared to the more slowly evolving cox 1 [28]. These authors were cautious in this regard, again reinforcing the need for more data.

Other than MG458326 (Eudiplozoon sp.), there are two sequences for which full identifications are not noted and for which some additional information may be deduced. MG458326 was noted as Sindiplozoon sp. and collected

Table 3 Sequence divergence (average uncorrected p-distance in \%) for diplozoid taxa based on partial cox1 mtDNA

\begin{tabular}{|c|c|c|c|c|c|c|c|c|c|c|c|c|c|c|}
\hline & GenBank ID & Species & 1 & 2 & 3 & 4 & 5 & 6 & 7 & 8 & 9 & 10 & 11 & 12 \\
\hline 1 & LC517176 & Eudiplozoon nipponicum & - & & & & & & & & & & & \\
\hline 2 & LC517177 & Eudiplozoon nipponicum & 0.51 & - & & & & & & & & & & \\
\hline 3 & AY009163 & Eudiplozoon kamegaii & 2.24 & 2.24 & - & & & & & & & & & \\
\hline 4 & LC517173 & Eudiplozoon nipponicum & 13.82 & 13.38 & 13.41 & - & & & & & & & & \\
\hline 5 & LC517174 & Eudiplozoon kamegaii & 14.25 & 13.67 & 13.45 & 0.24 & - & & & & & & & \\
\hline 6 & LC517175 & Eudiplozoon kamegaii & 14.25 & 13.01 & 13.17 & 3.65 & 3.21 & - & & & & & & \\
\hline 7 & MG458328 & Eudiplozoon sp. & 15.78 & 15.19 & 15.69 & 10.95 & 11.16 & 9.36 & - & & & & & \\
\hline 8 & MG458326 & Sindiplozoon sp. & 19.59 & 18.82 & 19.05 & 18 & 18.45 & 17.81 & 17.76 & - & & & & \\
\hline 9 & HF565162 & Paradiplozoon sp. ${ }^{a}$ & 20.94 & 20.62 & 20.17 & 20.39 & 19.59 & 20 & 20.6 & 18.36 & - & & & \\
\hline 10 & MG458327 & Paradiplozoon opsariichthydis & 21.63 & 19.73 & 20.45 & 23.11 & 22.78 & 22.6 & 22.37 & 22.59 & 20.35 & - & & \\
\hline 11 & KP399595 & Paradiplozoon homoion & 21.34 & 21.46 & 22.41 & 23.94 & 23.74 & 23.74 & 23.99 & 21.72 & 23.28 & 25 & - & \\
\hline 12 & KP399596 & Paradiplozoon gracile & 21.59 & 21.72 & 22.41 & 23.94 & 23.74 & 23.74 & 23.99 & 21.72 & 23.28 & 25 & 0.76 & - \\
\hline 13 & KP340976 & Diplozoon paradoxum & 22.88 & 23.23 & 22.97 & 21.01 & 21.21 & 22.98 & 23.23 & 22.73 & 20.63 & 24.49 & 22.22 & 22.47 \\
\hline
\end{tabular}

${ }^{a}$ This sequence represents Paradiplozoon ichthyoxanthon Avenant-Oldewage 2014 in Avenant-Oldewage et al. [41] and should be noted as such from here on out. 
from Spinibarbus hollandi Oshima, 1919, Parabramis pekinensis (Basilewsky, 1855) and Mylopharyngodon piceus (Richardson, 1846). Using the hosts, it is most probable that the species is Sindiplozoon diplodiscus (Nagibina, 1965) as this species has been recorded from M. piceus [3, 39]. However, it could also be Paradiplozoon parabramisi (Ling, 1973) which has been recorded from $P$. pekinensis $[3,40]$, although this would be unlikely due to the large p-distance between MG458326 and MG458327 (Paradiplozoon opsariichthydis (Jiang et al., 1984)), with both P. parabramisi and P. opsariichthydis forming part of the 'Paradiplozoon hemiculteri-complex' based on ITS2 rDNA, which will be discussed further on. To our knowledge, no diplozoid has been recorded and fully identified from $S$. hollandi, and thus this may either be a new host for either of the aforementioned diplozoid species, or indicate that the specimens in question represent a new species. The other sequence not fully identified is HF565162 for Paradiplozoon sp. This sequence was produced by the present authors during the description of Paradiplozoon ichthyoxanthon Avenant-Oldewage, 2014 in Avenant-Oldewage et al. [41] and prematurely uploaded. This sequence is thus representative of $P$. ichthyoxanthon and can be considered the cox $1 \mathrm{mtDNA}$ paragenetype for the species. The present publication will be used to update its GenBank record to avoid future confusion (captured in Table 3 and Additional file 1: Table S1).

\section{ITS2 rDNA}

Diplozoid sequences containing ITS2 rDNA fragments range from $577 \mathrm{bp}$ (KT781100 (unpublished and unidentified)) to 1762 bp (KY290757-KY290761 (P. hemiculteri, unpublished)), depending on the primers used. All available ITS2 sequence data were compiled, primers removed where required, $5.8 \mathrm{~S}$ and $28 \mathrm{~S}$ ends trimmed (based on binding regions of primers D and B1 [42]), and aligned using MAFFT [43] (via EBI) to remove subjectivity. The produced alignment contained 116 sequences and consisted of $814 \mathrm{bp}$, with 225 conserved, 569 variable, and
469 parsimony informative sites. Distances of up to $39.72 \%$ can be seen between diplozoid taxa, while many sequences for distinct taxa are identical or have very little variation (Additional file 2: Table S2). In Table 4, the pairwise distances between the six diplozoid genera (intergeneric) for which ITS2 data are available are given alongside their intrageneric distances. The intrageneric distance of Inustiatus is very low $(0.42 \%)$, but the two taxa designated by Gao et al. [40], Inustiatus inustiatus (Nagibina, 1965) and Inustiatus aristichthysi (Ling, 1973), were synonymised by Khotenovsky [3] in 1985, effectively producing a monotypic genus. Thus, the intrageneric distance of Inustiatus represents the intraspecific distance for I. inustiatus as well. This means that ITS2 sequence data for Sindiplozoon, Afrodiplozoon and Inustiatus are each only represented by a single taxon. Eudiplozoon also has a low intrageneric distance, but this distance represents two taxa, E. nipponicum and E. kamegaii, with intraspecific distances of up to $0.96 \%$ and an interspecific distance of 3.09-3.9\%, supporting the distinctness of these two species. Sequence data for these genera (Inustiatus, Eudiplozoon, Sindiplozoon and Afrodiplozoon) have plausible intraspecific distances $(<1 \%)$, indicating that all five taxa involved are likely distinct. Additionally, the intrageneric range of Eudiplozoon is far lower than the lower limits of the intergeneric distances for these four genera $(>20 \%)$, possibly confirming the genetic validity of these genera. On the other hand, the low intergeneric distance between Diplozoon and Paradiplozoon may indicate the opposite. Additionally, the high upper limit of the intrageneric distance for Paradiplozoon is concerning as this is higher than the lower intergeneric limits between all genera.

Within Paradiplozoon, intraspecific distances of $0-3.05 \%$ can be seen, while interspecific distance of as low as $0 \%$ are also seen (Additional file 2: Table S2). This suggests that there is something amiss with either the available data, the identification of specimens, or the viability of ITS2 rDNA to differentiate diplozoid species, and thus needs to be reassessed. There are two groupings

Table 4 Inter- and intrageneric sequence divergence (average uncorrected p-distance in \%) based on ITS2 rDNA

\begin{tabular}{|c|c|c|c|c|c|c|}
\hline & Diplozoon & Paradiplozoon & Sindiplozoon & Eudiplozoon & Inustiatus & Afrodiplozoon \\
\hline Diplozoon & $0-15.42$ & & & & & \\
\hline Paradiplozoon & $4.34-39.72$ & $0-39.61$ & & & & \\
\hline Sindiplozoon & $15.47-22.83$ & $15.44-36.3$ & a & & & \\
\hline Eudiplozoon & $19.26-27.87$ & $18.76-37.57$ & $20.52-24.47$ & $0-3.9$ & & \\
\hline Inustiatus & $25.25-32.05$ & $25.04-38.86$ & 25.57 & $22.49-27.11$ & 0.42 & \\
\hline Afrodiplozoon & $26.6-32.38$ & $13.49-32.55$ & 29.29 & $30.39-32.17$ & 32.83 & a \\
\hline
\end{tabular}

${ }^{a}$ No intrageneric distance possible as only a single sequence is available for genus.

Note: Intrageneric distance is indicated in bold 
of species for which interspecific distances of 0 were calculated, the 'P. homoion-complex' which may consist of three species occurring across Eurasia, and the 'P. hemiculteri-complex' which may consist of six species and occur in China. These two species complexes showed interspecific distances of $0-1.32 \%$ and $0-3.05 \%$ respectively and will be discussed in full further on. When these species-complexes are not considered, the intraspecific distances observed for Paradiplozoon become 0-0.44\%, while interspecific distances of $1.9-39.72 \%$ are calculated for the genus. These ranges do not overlap and as such a robust value of $1.5 \%$ can be used as the limit at which different diplozoid species can be differentiated using ITS2 rDNA. The upper intraspecific limit also compliments that seen in other diplozoid genera $(<1 \%)$, supporting this observation. If these limits are used to try and designate sequences which were not fully identified, sequences MN892630-MN892639 (unpublished) are clearly distinct from other diplozoid taxa (13.79-33.04\%, Additional file 2: Table S2) and thus represent a species not genetically typed using ITS2 before. Due to the lack of information in the GenBank records by Kadirden (Arken) and Cheng (Yue) for these sequences, and the fact that they are unpublished, we cannot infer much about their identity. Thus, the assumption of previous studies, that ITS2 rDNA may be a good marker for barcoding and delimitation of diplozoid species, may be correct and should continue, but only if closely related species complexes are disregarded or ideally resolved. However, the study of the evolutionary history of the Diplozoidae using this marker while species complexes are not yet fully resolved is not ideal.

\section{Phylogenetic studies}

To produce more accurate phylogenetic analyses of the ITS2 rDNA fragment, an outgroup was added to the alignment of diplozoid taxa, Neoheterobothrium hirame Ogawa, 1999 (FJ480941), as selected using BLASTn [44]. Analyses of the ITS2 alignment to produce an updated evolutionary history of the Diplozoidae was done using MEGA 7 and the BEAST v2.5.0 [45] software package. In MEGA 7, maximum likelihood (ML) and parsimony (P) methods were employed to reconstruct the evolutionary history. For ML, the general time reversible (GTR) model [46] with gamma distribution $(+\mathrm{G}$, parameter $=$ 2. 2524) was selected using the Model Selection Tool in MEGA 7. The robustness of both ML and MP analyses were tested with 1000 bootstrap replicates. Using BEAST v2.5.0, Bayesian inference (BI) analyses were performed, using 10 million Markov chain Monte Carlo (MCMC) generations. Using all generated topologies, a combined and updated topology based on BI analyses of the family was produced (Fig. 1) with MCMC and bootstrap support indicated at the respective nodes (BI/ML/P). Nodes with less than $50 \%$ support for all methods are not annotated, with those that have less than $50 \%$ support in certain analyses indicated with "-". Nodes not present in either ML or P topologies are indicated with “*”.

Jirsová et al. [32] and Fan et al. [31] produced the most recent and complete phylogeny of diplozoid parasites, which do not differ markedly when compared to the revised topology presented here (Fig. 1). Looking at the produced topology from the most basal taxa, it would appear that all diplozoid taxa are spilt into two major clades, indicated on Fig. 1 as Clade 1 and Clade 2. However, this grouping was only well supported by BI analyses. In both ML and P analyses Inustiatus and Eudiplozoon are consecutively basal to all other diplozoids, but the nodes for this were not well supported and mostly collapse at $50 \%$ consensus. The distant relation of E. nipponicum (or E. kamegaii depending on the host [22]) in both larval development [47] and molecular data to other diplozoids, prompted its use as an outgroup in several phylogenetic studies of the family [4, 29, 35, 36, 41, 48-50]. Whereas, Gao et al. [40] designated $N$. hirame of the Diclidophoridae as an outgroup in their analyses to produce a more accurate phylogeny, as was done presently. This concept was also utilised by Civáňová et al. [30] and Dos Santos \& Avenant-Oldewage [51] in designating Zeuxapta seriolae (Meserve, 1938) from the related Axinidae as an outgroup. Based on the topology presented here, even though BI analyses place Inustiatus and Eudiplozoon in Clade 1, the contradiction of this by ML and P methods mean that the placement of these genera are not resolved as of yet and their use as outgroups should be avoided. However, the grouping of Sindiplozoon with Paradiplozoon and Diplozoon taxa in Clade 1 is consistent across all methods used, with fairly high statistical support. Most nodes within the rest of this clade are also well supported, except for nodes related to the same taxa or those in species-complexes. Clade 1 contains roughly half of the Paradiplozoon and all the Diplozoon taxa for which sequence data are available.

The second major clade of diplozoid species, or Clade 2, consists of the single sequence for Afrodiplozoon and the other half of Paradiplozoon taxa. Interestingly, the present authors speculated that Afrodiplozoon may have proved an ideal outgroup to the Diplozoinae as it is currently in the subfamily Neodiplozoinae, but that does not seem to be the case based on ITS2 rDNA. Afrodiplozoon groups sister to Paradiplozoon spp. from Africa and Turkey, all of which form a sister clade to Asian (Chinese and Malaysian) Paradiplozoon spp., placing Afrodiplo$z o o n$ in the ingroup of Clade 2. The statistical support for the entire Clade 2 is fairly high and consistent across the three phylogenetic methods used, with the exception 


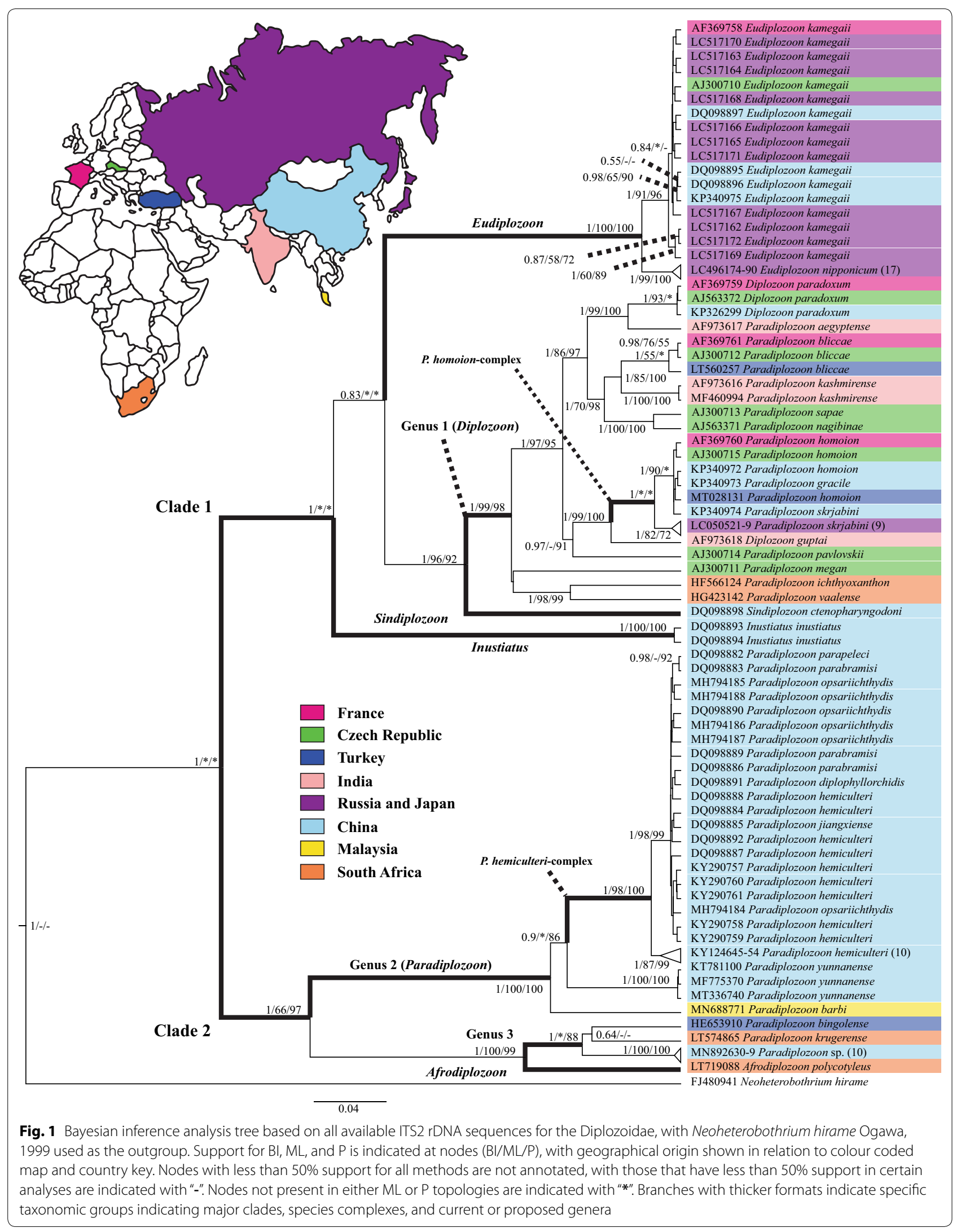


of nodes related to the same taxa or those in species complexes.

Looking at the discriminatory power of the ITS2 fragment based on the produced topology (Fig. 1), it would appear that the separation of most distinct taxa is well supported. The exceptions are the three Paradiplozoon taxa in Clade 1 which form part of the 'P. homoion-complex', and the six Paradiplozoon taxa in Clade 2 which represent the ' $P$. hemiculteri-complex', both of which will be discussed further on. To further assess the phylogenetic virility of the ITS2 marker, saturation of the aligned sequences was assessed using DAMBE 6.4.76 [52, 53], where the number of transitions and transversions were plotted against sequence divergence values (Fig. 2). From this plot, it is clear that the transitions of the ITS2 alignment became saturated. This may indicate that this marker is not ideal for inferring phylogenies, similar to what was seen for $\operatorname{cox} 1$ by Jovelin \& Justine [18]. Thus, the suitability of other markers to study cryptic groups like the Diplozoidae need to be more vigorously investigated. Unfortunately, due to the limited number of sequences available for the other markers discussed here, topologies and saturation plots of their alignments did not show any informative results and thus much more sequence data are needed for all these markers before meaningful comparisons can be made to the suitability of ITS2 rDNA for diplozoid taxonomy.

\section{Genus designation based on genetic analyses}

Based on the topology generated using ITS2 rDNA (Fig. 1), a few possibilities are evident in terms of the designation of genera in the Diplozoidae. First, that the entire group is monophyletic and that the Diplozoidae are monogenetic with only a single genus (Diplozoon). This is unlikely due to the vast genetic distances (Table 4, Additional file 2: Table S2) and morphological differences $[3,39]$ between current genera. Additionally, this would mean that the subfamilies Diplozoinae and Neodiplozoinae are invalid, although this is supported by the placement of Afrodiplozoon in Clade 2. Secondly, that species designated to Paradiplozoon represents two, three, or more genera. The second option is well supported by the topology and genetic distances produced here. If this were the case, one could effectively consider the grouping of Paradiplozoon and Diplozoon species in Clade 1 as Genus 1 (which would revert to the type genus Diplozoon), and the Paradiplozoon species in Clade 2 as Genus 2 (which would remain Paradiplozoon). The consistent grouping of D. paradoxum within certain Paradiplozoon taxa, separate from other Paradiplozoon taxa (Clade 2), has lead authors to speculate the paraphyly of Paradiplozoon, highlighting the need for a taxonomic revision of the group [30, 48, 49]. This, alongside the updated topology and the grouping of Diplozoon guptai Fayaz \& Chishti, 2000 with Paradiplozoon species in Clade 1, but not sister to D. paradoxum, may support the notion that Paradiplozoon and Diplozoon in Clade 1 are monophyletic. This idea, and the subsequent notion that Diplozoon and Paradiplozoon species should all be considered Diplozoon, was already proposed by Sicard et al. [48], but was never applied to later studies by other authors. However, we now know that the general synonymising of these two genera was premature as there are a substantial number

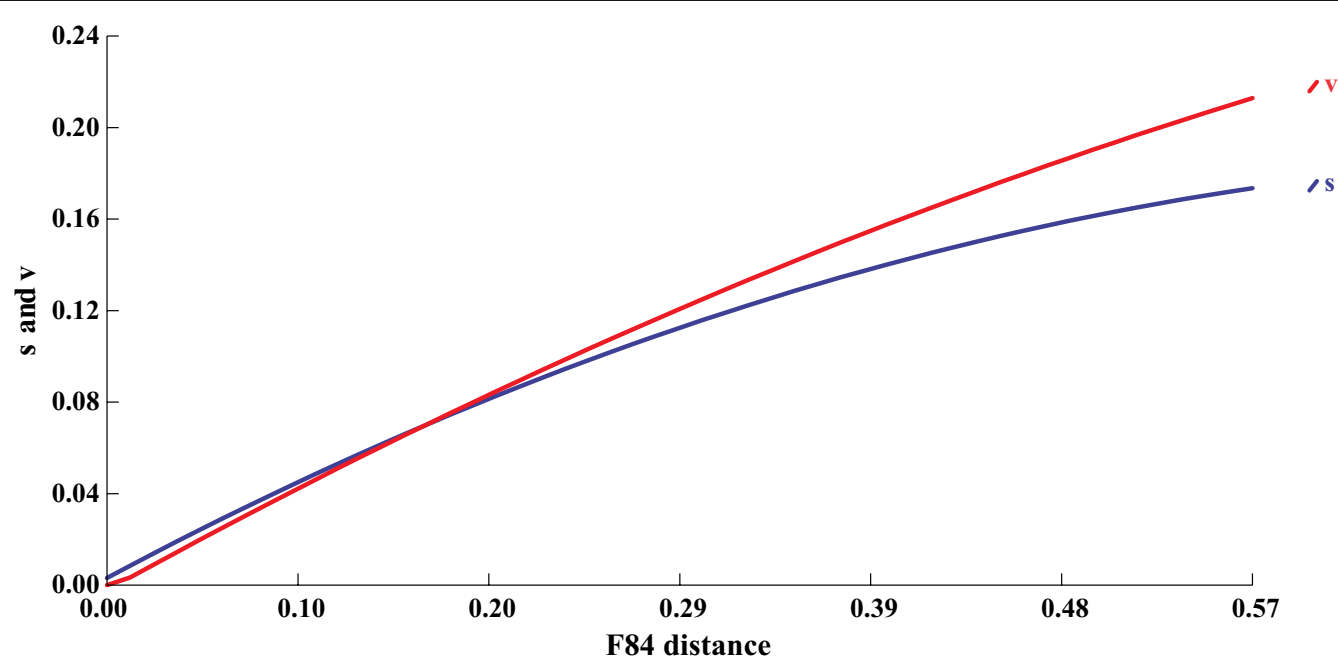

Fig. 2 Saturation plot of the number of transitions ( $\mathrm{s}$, blue) and transversions ( $\mathrm{V}$, red) plotted against sequence divergence values (F84 distance) of the alignment of all available ITS2 rDNA sequences for the Diplozoidae 
of Paradiplozoon species in Clade 2 which are clearly not monophyletic to the species included in Diplozoon by Sicard et al. [48].

Due to the grouping of Afrodiplozoon within the ingroup of Clade 2, the designation of a third genus might be possible, with Genus 2 sister to a grouping of Afrodiplozoon and Genus 3. This implied change in the taxonomic designation of groups will also be problematic when considering the current taxonomy of the Diplozoidae. There is currently no robust morphological criteria on which to distinguish the members of Genus 1 (Diplozoon), 2 (Paradiplozoon), and 3 confidently. And again, as Afrodiplozoon (Neodiplozoinae) is in the ingroup of Clade 2 (Diplozoinae), the designation of subfamilies will also need to change or fall away. It would appear that according to ITS2 rDNA, the division of diplozoid genera into two subfamilies based on the number of clamp pairs [3,39] might be entirely incorrect. If we were to use these new designations of genera and apply them to the genetic distances calculated for available sequence data, the inter- and intrageneric distances as shown in Table 5 are produced. From the distances captured in Table 5, it is clear that the limits between the newly proposed designation of genera are more separate, but still overlap slightly. The high intrageneric distance of Genus 1 (Diplozoon) and low intergeneric distance between Genus 2 (Paradiplozoon) and Afrodiplozoon show that no concrete limits for the designation of genera can be seen at this time using ITS2 rDNA. However, it should be made abundantly clear that these are purely speculative designations (summarised in Additional file 2: Table S3) based only on the topology and distances of ITS2 rDNA sequences presented here. This needs to be intently studied using other markers and rigorous morphological analyses, also possibly with co-phylogenetic studies alongside host material, of all genera before being considering viable. This could prove a very interesting endeavour and may re-shape the way we think about the taxonomy of cryptic groups such as the Diplozoidae.

\section{Phylogeography}

In an attempt to determine if any phylogeographic patterns can be seen from the topology produced using ITS2 rDNA, the geographical origin of the specimens used to produce respective sequence data have been indicated on the topology of Fig. 1. As can be seen from the topology (and Additional file 1: Table S1), two genera (Inustiatus and Sindiplozoon) occur solely in Asia (China), while another occurs only in Africa (Afrodiplozoon). The remaining genera occur across Eurasia, with Paradiplozoon also occurring in Africa. Specimens of E. kamegaii included in molecular studies were collected from Turkey, France, the Czech Republic, Japan, and China, but were all collected from $C$. carpio. These cyprinids are indigenous to Asia, presumably introduced to Europe [54], allowing E. kamegaii to spread alongside its host. Similarly, D. paradoxum was collected and sequenced from France, the Czech Republic, Sweden (based on the above analysis of $28 \mathrm{~S}$ rDNA sequence AF131717), and China from the type-host Abramis brama (Linnaeus, 1758). This linkage between the distribution of diplozoid parasites and their type-hosts is likely due to the direct life-cycle of monogenean parasites, enabling anthropogenic distribution.

Paradiplozoon species on the other hand appear to have a wide distribution across Eurasia and Africa. However, some observations can be made regarding the phylogeographic properties of this genus. All Paradiplozoon specimens collected from Europe are included in Clade 1 alongside all species of Diplozoon. However, there are also specimens collected from Asia and Africa in this clade. The close affiliation between European Paradiplozoon species and Diplozoon taxa has been shown to mirror the close relationship of their hosts $[4,29,48$, 49], further supporting the idea that all of these species belong to a single genus (Genus 1 (Diplozoon) as

Table 5 Inter- and intrageneric sequence divergence (average uncorrected p-distance in \%) based on ITS2 rDNA following suggested genus designation

\begin{tabular}{|c|c|c|c|c|c|c|c|}
\hline & Genus 1 (Diplozoon) & Genus 2 (Paradiplozoon) & Genus 3 & Sindiplozoon & Eudiplozoon & Inustiatus & Afrodiplozoon \\
\hline Genus 1 (Diplozoon) & $0-25.08$ & & & & & & \\
\hline Genus 2 (Paradiplozoon) & $29.46-39.72$ & $0-14.61$ & & & & & \\
\hline Genus 3 & $24.93-35.18$ & $25.77-32.69$ & $0-9.67$ & & & & \\
\hline Sindiplozoon & $15.44-22.88$ & $30.83-36.3$ & $26.29-27.17$ & a & & & \\
\hline Eudiplozoon & $18.76-28.19$ & $30.04-37.57$ & $27.69-33.55$ & $20.52-24.47$ & $0-3.9$ & & \\
\hline Inustiatus & $25.04-32.05$ & $32.66-38.86$ & $30.03-31.13$ & 25.57 & $22.49-27.11$ & 0.42 & \\
\hline Afrodiplozoon & $25.97-32.38$ & 29.69-32.55 & $13.49-15.44$ & 29.29 & $30.39-32.17$ & 32.83 & a \\
\hline
\end{tabular}

a No intrageneric distance possible as only a single sequence is available for genus.

Note: Intrageneric distance is indicated in bold 
discussed before). However, from a geographical origin, members of this group are widely spread and the river systems in which they occur do not share a common ocean or region.

Considering the Paradiplozoon species in Clade 2 (Fig. 1), we can see that most species are from Asian (China and Turkey) origin, except for Paradiplozoon krugerense Dos Santos \& Avenant-Oldewage, 2016 from Africa and the two species for which no locality data are currently available (Paradiplozoon barbi (ReichenbachKlinke, 1951) and Paradiplozoon sp.). For the latter two species, $P$. barbi has only been recoded from Malaysia and the sequence authors are based in Malaysia, thus it can be assumed that the specimens were collected from this country. Similarly, the authors of the unidentified Paradiplozoon sp. are based in China, making it highly probable that both these taxa are of Asian origin. When the Asian and African characters of the Paradiplozoon species in Clade 2 are considered, in conjunction with the possibility of two additional diplozoid genera within this clade as discussed before (Genus 2 (Paradiplozoon) and Genus 3), a possible integrated picture emerges. Genus 2 (Paradiplozoon) is then entirely of China-Malaysia origin, consisting of P. barbi, P. yunnanense and members of the 'P. hemiculteri-complex. This could mean that the ancestor to these species may have originated in the South China Sea or other parts of the Pacific Ocean. Genus 3 would then consist of Paradiplozoon species collected from Africa, Turkey and China. As both P. krugerense and Paradiplozoon bingolense Civáňová et al., 2013 were collected from river systems which eventually enter the Indian Ocean, this may indicate that members of this proposed genus have an Indo-Pacific ancestor, further supported by the close phylogenetic relation to the members of Genus 2. Collection of A. polycotyleus also took place from a river system entering the Indian Ocean, supporting the possible Indo-Pacific origin of all species in Clade 2. An appropriate designation for Genus 3 may then be Indodiplozoon based on the possibility of an ancestor from the Indo-Pacific (Additional file 2: Table S3).

\section{Species complexes}

\section{Paradiplozoon homoion-complex}

From the results presented here (Additional file 2: Table S2, Fig. 1), three species of diplozoids in Clade 1 are very closely related when considering the ITS2 rDNA fragment. These are P. homoion, P. gracile, and Paradiplozoon skrjabini (Akhmerov, 1974). Paradiplozoon homoion was originally described from Rutilus rutilus (Linnaeus, 1758), but has since been collected from more than 30 species of cyprinids, displaying uncharacteristically loose host specificity [55]. The identity of $P$. gracile has changed several times since it was originally described as Diplozoon gracile Reichenbach-Klinke, 1961, being reclassified as Diplozoon homoion gracile Oliver \& ReichenbachKlinke, 1973 (supported by Prost [56]), then moved to the genus Paradiplozoon as Paradiplozoon homoion gracile (Reichenbach-Klinke, 1961), and currently known as $P$. gracile. As can be seen from the shift of this species from a distinct taxon, to a subspecies, back to a standalone species, the validity of this species has been the topic of some debate. Most of the aforementioned taxonomic changes were based on morphological differences and similarities observed between $P$. gracile and P. homoion. Using allozyme analysis, Le Brun et al. [57] suggested that these species could be differentiated, while both Sicard et al. [29] and Matejusová et al. [4] concluded $P$. gracile a synonym for $P$. homoion based on identical ITS2 rDNA even when collected from a range of hosts. Matejusová et al. [55] attempted to revise the relation of these species, referring to them as the 'P. homoioncomplex' and concluded that $P$. gracile should be considered a species inquirenda as they were unable to detect a robust criterion for distinguishing the two supposed taxa based on detailed sclerite morphology and ITS2 sequences. As such, most studies in which P. gracile was studied using molecular tools did not publish separate sequence data as they were identical to those obtained for $P$. homoion. However, two sequences have been made public through GenBank by Wang \& Yue (unpublished) for the $\operatorname{cox} 1$ and ITS2 markers of P. gracile. For the ITS2 rDNA fragment assessed above, $P$. homoion and $P$. gracile sequences are identical, while $0.76 \%$ separate representative sequences of these species when cox $1 \mathrm{mtDNA}$ is considered. This supports the findings of other studies indicating no resolution between members of this species complex using ITS2 rDNA, but may showcase the ability of cox $1 \mathrm{mtDNA}$ in differentiating intrinsically close taxa. This may be supported by the idea that the allozyme difference between $P$. homoion and $P$. gracile indicate local partial genetic differentiation, but that there is either still enough gene flow between them to maintain rDNA similarity, or that their separation is due to a recent barrier and thus sequence variation is delayed [48].

Looking at the produced topology and the calculated distances using ITS2 rDNA fragments (Additional file 2: Table S2, Fig. 1), P. skrjabini collected in Russia, Japan, and China [58] are also closely related to the widely distributed P. homoion. Similar to P. homoion and P. gracile, $P$. skrjabini has also been collected from several host species (7 to date), and thus shows relaxed host specificity. According to Shimazu et al. [58], P. skrjabini is morphologically very similar to $P$. homoion, only differing by the absence of anterior joining sclerites in the latter species. These three species form a well-supported 
clade, with most of the sequences for P. skrjabini sister to those of $P$. homoion, $P$. gracile and a single sequence for P. skrjabini, the two groupings separated by $1.02-1.32 \%$. The sequence for P. skrjabini (KP340974) which groups with $P$. homoion and $P$. gracile is yet unpublished, but is noted from the same host genus as sequence LC050528 (P. skrjabini from Leuciscus waleckii (Dybowski, 1869)). Also, KP340974 is only separated from sequences for $P$. homoion and $P$. gracile by $0.3 \%$, while it is separated from other P. skrjabini sequences by 1.17-1.32\%. From these results, two possibilities arise. First, that $P$. skrjabini also forms part of the 'P. homoion-complex', bringing the total number of species in the complex to three, with intraspecific distances of up to $1.32 \%$ based on ITS2 rDNA. This is supported by the $1.5 \%$ robust limit designated earlier to discriminate between species based on ITS2 rDNA. The second possibility would be that the identification of KP340974 was incorrect and truly represented either $P$. homoion or P. gracile. This would mean that the ' $P$. homoion-complex' consists only of $P$. homoion and $P$. gracile, with $P$. skrjabini a very closely related sister taxon, possibly indicating a subspecies. The latter scenario is supported by Khotenovsky [3], who lists Leuciscus baicalensis (Dybowski, 1874) (from which KP340974 (P. skrjabini) were collected) as a host of P. homoion. However, when considering hosts, both $P$. homoion and P. skrjabini parasitize members of the genus Leuciscus, while all three diplozoid species have been recorded from Phoxinus hosts. Additionally, Sicard et al. [48] analysed an unidentified diplozoid parasite collected from Tribolodon hakonensis (Günther, 1877) in Japan, the same host and locality from which diplozoids were collected and identified as P. skrjabini by Shimazu et al. [58]. Sicard et al. [48] recorded a close molecular relationship of these parasites from $T$. hakonensis to $P$. homoion collected in Europe, similar to what is seen in the generated topology (Fig. 1), suggesting that they were in fact studying P. skrjabini. Unfortunately, this sequence data were not published and are unavailable for analyses.

It is thus most likely that all three the species in question form part of the ' $P$. homoion-complex' coined by Matejusová et al. [55]. The wide geographical distribution of this cryptic 'P. homoion-complex' may even reach into Africa as Sicard et al. [48] noted that sequences obtained for diplozoid parasites collected from Labeo coubie Rüppell, 1832 in Ivory Coast were genetically "strictly similar" (sic.) to those of P. homoion collected in Europe. Unfortunately, this sequence data were also not published, and as such the data cannot be analysed. It is thus necessary to study representatives of all the diplozoids which could be attributed to these three species in order to further elucidate their taxonomic status and that of the entire species complex. In this regard, the use of additional markers, like cox $1 \mathrm{mtDNA}$ (which appears to enable discrimination between $P$. homoion and $P$. gracile unlike ITS2 rDNA), would be informative. Newly implemented morphometric techniques, such as the study of diplozoid sclerites using scanning electron microscopy (SEM) [59, 60], may also be useful in this regard.

\section{Paradiplozoon hemiculteri-complex}

From the results presented here (Additional file 2: Table S2, Fig. 1), six species of diplozoids in Clade 2 are very closely related when considering ITS2 rDNA, forming a purely Chinese Paradiplozoon clade. These are $P$. hemiculteri, P. opsariichthydis, P. parabramisi, Paradiplozoon diplophyllorchidis (Jiang et al., 1985), Paradiplozoon parapeleci (Jiang et al., 1984) and Paradiplozoon jiangxiense (Jiang et al., 1985). All these diplozoids were collected in adjacent river basins, the Yangtze and Pearl River Basins, but from a number of different host species. Sequences for all these species form a well-supported clade, with a grouping of select sequences for $P$. hemiculteri sister to a grouping of the remaining $P$. hemiculteri sequences and sequences of the other five species. The first grouping contains only $P$. hemiculteri sequences produced from a single study [32] and all from the same host, Hemiculter leucisculus (Basilewsky, 1855), the type-host of the species, with intraspecific distances of $0-2.34 \%$. Sequences in the second grouping (containing all six species in question), were produced during several independent studies, some of which are unpublished, and from seven hosts including $H$. leucisculus, with intraspecific distances of $0-0.66 \%$. Between these two groupings, $1.71-3.05 \%$ uncorrected p-distances were calculated, with the highest values between sequences both identified as P. hemiculteri (DQ098892 and KY124652) and collected from the same host, indicating that there is something amiss regarding the sequence data in this clade. Jirsová et al. [32], the authors for the sequences of the first grouping containing only $P$. hemiculteri, noted that the divergence between the sequences in their study were negligible, with only $0.1-0.3 \%$ separating them. But the data produced here (Additional file 2: Table S2), which were generated using data retrieved from GenBank, contradict this with values up to $2.34 \%$, which is much higher than the $1.5 \%$ robust limit designated earlier to discriminate between species based on ITS2 rDNA. However, if this robust limit of $1.5 \%$ is used to consider the two groupings, the second grouping within the Chinese Paradiplozoon clade should represent a distinct taxon, as it is separated from the first grouping by $1.71-3.05 \%$.

As such, two possibilities arise. First, that all six Paradiplozoon species in this clade represent members of a ' $P$. hemiculteri-complex' which has a very large sequence 
divergence of up to $3.05 \%$ for ITS2 rDNA. This does not seem likely, as this would call into question the validity of all diplozoid species that are separated by less than $3.05 \%$ ITS2 rDNA sequence divergence. Secondly, that some form of error has occurred in the data capturing of the Paradiplozoon sequences from China. Jirsová et al. [32] noted that their collections took place at the type-locality of $P$. hemiculteri. Thus, it is fair to expect that their sequences do indeed represent $P$. hemiculteri from $H$. leucisculus. That implies that the identification of both $P$. hemiculteri and their hosts collected by Gao et al. [40] and $\mathrm{Xi}$, Buga \& Li (unpublished) may be incorrect [32]. In this case, there may be two species complexes in China, a ' $P$. hemiculteri-complex' in the Pearl River Basin (based on the high divergence of the sequences of Jirsová et al. [32]), and a 'Paradiplozoon parabramisi-complex' in the Yangtze River Basin (P. opsariichthydis, P. parabramisi, $P$. diplophyllorchidis, $P$. parapeleci, $P$. jiangxiense and $P$. hemiculteri sequences by Gao et al. [40] and Xi, Buga \& Li (unpublished)). Jirsová et al. [32] noted that morphological descriptions of Chinese diplozoids presented a hinderance to their study, with the inaccurate identification and lack of morphological records in genetic studies compounding the issue. Again, the use of additional markers like cox1 mtDNA alongside expanded morphometric analyses $[59,60]$ may be informative to resolve this question.

\section{Species identification hurdles}

Recently, three diplozoid species were genetically characterised from India, producing the first sequence data for diplozoid parasites from the subcontinent $[35,36]$. Two of these species, D. guptai and Paradiplozoon kashmirense (Kaw, 1950) have been described and recorded from the subcontinent and its native fish. But the third species, Paradiplozoon aegyptense (Fischthal \& Kuntz, 1963) was described and previously recorded only from Africa and native African fish. This species was collected from indigenous hosts in India and was distinct from all other diplozoid species by at least 5.62\% (ITS2 rDNA). Thus, the diplozoids collected by Ahmad et al. [35, 36] from Carassius carassius (Linnaeus, 1758) and Schizopyge niger (Heckel, 1838) represent a diplozoid taxon not previously sequenced and little doubt exists about its genetic distinctness. However, due to the outdated literature used by the authors to identify the diplozoid taxa in their study, still referring to the species as Diplozoon aegyptensis, in addition to $P$. aegyptense only recorded from native African cyprinids in the past, the identification of these diplozoids as $P$. aegyptense is unconvincing. This is further complicated by the findings of Sicard et al. [48] as mentioned above, where diplozoids collected from Ivory Coast were noted to be genetically "strictly similar" (sic.) to P. homoion. Sicard et al. [48] collected these specimens from L. coubie, a recorded host of $P$. aegyptense, unlike those of Ahmad et al. [35, 36]. As such, several possibilities arise. First, that P. aegyptense is a synonym for $P$. homoion based on the findings of Sicard et al. [48], and as such the identification by Ahmad et al. $[35,36]$ is incorrect as the obtained sequence is not similar to those for P. homoion. Secondly, that some form of human error occurred during the study of Sicard et al. [48] with sequences for P. homoion mistakenly attributed to diplozoids from the Ivory Coast, allowing for the possibility that the identification by Ahmad et al. [35, 36] was correct. Finally, it is also possible that $P$. homoion can infect native African fish hosts and that the diplozoids collected from $L$. coubie by Paperna $[7,8]$ were in fact $P$. homoion. This would mean that the diplozoids studied by Sicard et al. [48] do not truly represented P. aegyptense. Without genetic characterisation of diplozoids collected from the type-host in the type-locality to confirm the status of $P$. aegyptense, this mystery persists. Additionally, $P$. kashmirense has been noted as a synonym of E. nipponicum [61] (possibly E. kamegaii [22]). Given the distinct nature of the sequences currently available for P. kashmirense and their large distance from those for E. nipponicum, either the identification or the synonymizing with $E$. nipponicum was incorrect. This suggests that much work is still needed to fully incorporate diplozoid taxa from the subcontinent in taxonomic and systematic studies.

Another example of problematic species identification can be seen with the diplozoid specimens studied by Zhang et al. [28]. Eudiplozoon specimens were collected from C. auratus in China and may thus represent E. nipponicum which was recently confirmed to infect Carassius sp. in Japan [22]. Zhang et al. [28] produced ITS2 rDNA sequence data for these worms and note that they differed by $5-6 \%$ from E. kamegaii. Unfortunately, they did not publish these sequences and thus cannot be directly compared to the recently published sequence data for E. nipponicum. Fortunately, cox $1 \mathrm{mtDNA}$ could be mined from the mitogenomes published by Zhang et al. [28] and a comparison with both E. nipponicum and E. kamegaii was possible. Cox1 data for the unidentified species of Eudiplozoon were distinct from other cox1 sequences for the same genus (9.36-15.78\%). As discussed, the cox 1 data for E. nipponicum and E. kamegaii do not provide clear taxonomic results, with either two or four taxa possible. Nevertheless, the large distance of the data produced by Zhang et al. [28] from other Eudiplozoon cox 1 data, alongside the large ITS2 distance from $E$. kamegaii (which is almost double the distance between $E$. kamegaii and E. nipponicum), suggest that the specimens do not represent E. nipponicum, but instead a cryptic, possibly undescribed species of Eudiplozoon as suggested 
by Zhang et al. [28]. Interestingly, Sicard et al. [48] also collected a diplozoid from $C$. auratus (the same host as Zhang et al. [28]) in Japan (the same locality as Nishihira \& Urabe [22]) and noted it to be $2.74 \%$ (ITS2 rDNA) from E. kamegaii. The ITS2 distance, locality, and host of the samples by Sicard et al. [48] relate more closely to $E$. nipponicum [22] than the unidentified Eudiplozoon from Zhang et al. [28]. But again, the sequence data of Sicard et al. [48] were not published and cannot be critically assessed. The apparent dissimilarly between Eudiplozoon specimens from C. auratus in Japan and China is puzzling but may provide a good opportunity to study the morphometric and genetic variation between these hostparasite groups. This may also help resolve the apparent uncertainty around the identity of Carassius species in these areas as Nishihira \& Urabe [22] note the hosts of $E$. nipponicum they collected as C. buergeri grandoculis and Carassius sp. (common names nigorobuna and ginbuna, respectively), which are synonyms for $C$. auratus and Carassius langsdorfii Temminck \& Schlegel, 1846 respectively according to FishBase [62].

Similarly, Zhang et al. [28] collected an unidentified Sindiplozoon sp. from several host species in China, differing by $3 \%$ from available ITS 2 rDNA sequence data for this genus. The taxonomy of this genus is very understudied, with between two [3, 39] and six species [63] considered valid. The distance between published Sindiplozoon ITS2 sequence data and that produced by Zhang et al. [28], support that the latter does not represent Sindiplozoon ctenopharyngodoni (Ling, 1973) and instead a diplozoid not previously sequenced (most likely $S$. diplodiscus as discussed earlier). Like the sequence data for the unidentified Eudiplozoon sp., the ITS2 rDNA data for the unidentified Sindiplozoon sp. were not published and micrographs for taxonomically important structures of these samples included in the publication are not sufficient to accurately describe or identify the species. However, the fact that the ITS2 rDNA sequence data produced by Zhang et al. [28] for P. opsariichthydis were identical to previously published data for this species, confirms the credibility of their data and suggests the potential for at least one new species (Eudiplozoon) and the genetic characterisation of at least one cryptic species (Sindiplozoon). All the aforementioned circumstances highlight the importance of generating comprehensive, accurate, and integrated data that include morphological and genetic aspects for known taxa, as well as new and cryptic species, preferably from the type-hosts and localities to prevent unnecessary confusion. Additionally, the defining of a more accurate and robust system for the successful identification and differentiation of diplozoid species is sorely needed and should be actively pursued.

\section{Inconsistencies and ethics}

During the collection of data and material for this review, several inconsistencies and unconvincing notions were uncovered. Apart from those mentioned in the previous sections, there are some which relate more to the procedural and ethical aspects of molecular taxonomy which should also be addressed. The first is the use of outdated generic placement when referring to diplozoid species. To date, the most complete and widely accepted revision of the Diplozoidae was by Khotenovsky [3]. This work is difficult to source and was published in totality in Russian, with information for species occurring in only the Amur and Palaearctic regions translated and published in English by Pugachev et al. [36]. Thus, it is understandable that some authors are not aware of the revised taxonomy of some species. However, when Khotenovsky [3] is cited as part of the literature used in a study, and the new designation of the species is ignored without reason, it creates unnecessary confusion and uncertainty.

Another point of concern is the publication and release of sequence data. As mentioned, several sequences are currently available on GenBank even though they do not refer to any published work. This has both negative and positive aspects. On the one hand, such sequence data are available and can be included in the work of other researches to obtain larger data sets and often more comprehensive results. But having access to data which have not and possibly may never be peer-reviewed and published, often raises more questions than answers. The present authors acknowledge that this is an easy error as they are responsible for GenBank sequence HF565162 which was also not linked to a published work until now. An attempt to reconcile and complete missing or incomplete data was attempted here and, as per the previous sections, were explained and captured (Additional file 1: Table S1). However, some information for the more cryptic data could not be deduced, and one can only hope that the authors of those sequences will at some point publish their findings and make the information available.

Finally, it would appear that some studies have been published in duplicate in different journals, with identical sequence data designated different accession numbers. This creates unnecessary confusion as it is unclear whether these sequences are from separate individuals and can be used to infer intraspecific variation or stability, or are simply duplicates of the same data.

\section{Final suggestions and plea for voucher material}

As is clear from the points discussed and the molecular data reviewed, we have only scratched the surface of the molecular study of the Diplozoidae. However, by summarising, reviewing and scrutinising the available 
information, it can now be attempted to steer this endeavour in the right direction. The first point to consider is the information obtained from online data repositories. As has been illustrated here, the data obtained from such sources are not always complete or related to a published, peer-reviewed work. It is also clear that using some of the data may create more confusion than it resolves. As such, we propose that the merit and value of data be assessed before being included in analyses. In doing this, it should be possible to limit the confusion these sequences may cause. For example, the sequence for an unidentified Paradiplozoon sp. by Kadirden (Arken) and Cheng (Yue) (MN892630-MN892639) clearly represent a distinct diplozoid taxon and thus contain valuable information, but they have not been published and thus very little information can be gathered for them. This means that any conclusions drawn from them in the present manuscript are entirely speculative and cannot be considered anything else.

Another consideration is the proper study and cataloguing of morphological data. Looking at several of the papers mentioned here, morphological study of the specimens in question are glossed over. Whether this is because the authors are not familiar with diplozoid taxonomy, whether the morphology is neglected (as with many purely molecular studies), or because the addition of descriptive morphology would make the study too large, none of these scenarios are conducive to the holistic study of any organism. This is because the outcome is that sequence data often remain unidentified, some species are incorrectly identified, or that sequence data or supporting information are never properly published and lost. These outcomes force future studies to make speculative conclusions (as was done here) with little hope of reaching a satisfactory conclusion. They also make the revisiting of specimens and whole studies necessary to re-assess the morphology of the specimens in question, which is often impractical if not impossible, also ending with speculative and unsatisfactory conclusions. Data produced and discussed in dissertations, theses, and conference proceedings, but never actively peer-reviewed, published, or submitted to data repositories, are also usually not obtainable and do not allow re-examination. The only way to prevent these issues is to either accurately record the full taxonomic details of the specimens in the publication accompanying sequence data, or to provide voucher material which can be re-examined at a later stage, bringing us to the final point of discussion.

A prominent issue which has been made clear in this review is the lack of accurate historical data and the completeness thereof. Recent descriptions of diplozoid taxa have included not only the full morphological description of the taxa, but also representative sequence data for
ITS2 rDNA. This means that the sequence data for these species were likely obtained from the same cohort as the other taxonomic information used to describe the species and can thus be considered the holo- or paragenetype for the species [64]. Unfortunately, this is only the case for a handful of species [30, 31, 41, 50, 51], with all those produced before 2012 lacking genetic data for the typematerial. Naturally, one could not expect the description of $D$. paradoxum to contain genetic data in 1832, but this illustrates the need for those producing species data now to consider the future. This means that studies involving the collection and study of organisms should make contingency arrangements, as far as realistically possible, for the future re-examination of material. To this end, we propose the following suggestions: (i) that voucher material be designated whenever diplozoid specimens are collected; (ii) that voucher material consists of as many formats as possible (e.g. 70\% $\mathrm{EtOH}, 96 \% \mathrm{EtOH}, \mathrm{GAP}$ slides); (iii) that voucher material be housed in more than one location; (iv) that the housing institution/collection is freely accessible to researchers globally (longstanding and established museums); and (v) the location and accession codes of the voucher material be properly recorded in the relevant publication. Following these guidelines, it may be possible to overcome some of the hurdles exposed in the present review. For many of the studies mentioned, if vouchers were deposited in a museum collection and material could be re-examined with both morphological and molecular tools, their results may have been substantiated or revised, allowing for a more accurate taxonomic view of the Diplozoidae. These vouchers could then also be designated as genophore vouchers following Pleijel et al. [65], allowing for future genetic studies. Hologenophores (material for genetic study obtained from holotype specimen) and paragenophores (material for genetic study obtained from paratype specimens) [65] are not available for most diplozoids, even for species for which genetypes were included in their description, and thus if any other markers would need to be assessed for these species in the future, one would have to collect fresh material. These would then represent syngenophore (material for genetic study obtained from specimens of a specific species, but from a different occasion and/or locality) material, from which genetypes (neogenetype, topogenetypes, etc.) could be retrieved. The latter would be the only way to obtain genetic information of species described in the past as the submission of material fit for molecular analyses is not common for monogeneans. However, collecting new material allows for the designation of neotype material for species of which the original type material is missing or lost, like was done for $P$. hemiculteri [32] and E. nipponicum [22], but this is not always possible. 


\section{Conclusions}

In conclusion, it is clear that a substantial amount of morphological and genetic data are needed before an accurate study of the taxonomy and evolutionary history of diplozoid species can be achieved. But, by identifying the causes of confusion and uncertainty from currently available information, they can be avoided in future studies. An inclusive, multidisciplinary approach to such studies will need to be incorporated, alongside the proper collection and preservation of voucher material. This should include accurate morphological identification (using traditional as well as modern taxonomic techniques), the use of molecular techniques with various markers, and the proper deposition and curation of both morphological and genophore vouchers, all actively complementing each other. Additionally, the co-phylogenetic study of diplozoids and their hosts may add valuable insight into the taxonomy and evolution of both groups. Finally, by summarising the work that has been done and analysing the produced data in a wholistic manner, it is hoped that the present review will serve as a reference point for diplozoid study and promote integrative taxonomy in future endeavours.

\section{Supplementary information}

Supplementary information accompanies this paper at https://doi. org/10.1186/s13071-020-04417-3.

Additional file 1: Table S1. Table containing all sequence data available for the family Diplozoidae, as compiled from public data repositories (GenBank) and literature.

Additional file 2: Table S2. Sequence divergence (average uncorrected p-distance in \%) for diplozoid taxa based on ITS2 rDNA. Table S3. Proposed genus designation based on $\mathrm{p}$-distance and phylogenetic results.

\section{Abbreviations}

BI: Bayesian inference; BLASTn: Nucleotide Basic Local Alignment Search Tool; cox 1: cytochrome c oxidase subunit 1; EBI: European Bioinformatics Institute; GTR: general time reversible model; ITS1: first internal transcribed spacer region; ITS2: second internal transcribed spacer region; MCMC: Markov chain Monte Carlo generations; MAFFT: multiple alignment using fast Fourier transform; ML: maximum likelihood; mtDNA: mitochondrial DNA; P: parsimony; rDNA: ribosomal DNA; SEM: scanning electron microscopy.

\section{Acknowledgements}

The authors would like to thank Professor Bettine Jansen van Vuuren for her insight and assistance with regards to data analyses and interpretation of data.

\section{Authors' contributions}

The project was conceptualised and planned equally by both authors. Data compilation and analyses, as well as the first draft of the manuscript were produced by QMDS. The manuscript was finalised by QMDS and AA-O. All authors read and approved the final manuscript.

\section{Funding}

The authors would like to thank the University of Johannesburg for funding from the University of Johannesburg Faculty and Central Research Committee (AA-O) and the University of Johannesburg Global Excellence and Stature Postdoctoral Fellowship program (QMDS). The National Research Foundation
(NRF) of South Africa is also thanked for their financial support to AA-O Grant 115343.

\section{Availability of data and materials}

Data used in this review were obtained from online data repositories and published literature. No new data were generated. Alignments and other datasets composed in this review will be provided on request.

\section{Ethics approval and consent to participate}

Not applicable.

\section{Consent for publication}

Not applicable.

\section{Competing interests}

Not applicable.

Received: 8 July 2020 Accepted: 21 October 2020

Published online: 30 October 2020

\section{References}

1. Anderson RM. An analysis of the influence of host morphometric features on the population dynamics of Diplozoon paradoxum (Nordmann, 1832). J Anim Ecol. 1974;43:873-87.

2. Gläser HJ, Gläser B. Zur Taxonomie von Diplozoon Normann, 1832. Zeitschr Parasitenkd. 1964;25:164-92.

3. Khotenovsky IA. Suborder Octomacrinea Khotenovsky. In: Scarlato OA, editor. Fauna of the USSR. Monogenea. Moscow: Russian Academy of Sciences, Zoological Institute; 1985.

4. Matejusová I, Koubková B, D'Amelia S, Cunningham CO. Genetic characterization of six species of diplozoids (Monogenea; Diplozoidae). Parasitology. 2001;123:465-74.

5. Thomas JD. A new monogenetic Trematode, Diplozoon ghanense, sp. nov. (Polyopisthocotylea: Discocotylea) from the West African freshwater fish Alestes macrolepidotus (C. \& V., 1849), in West Africa. J West Afr Sci Assoc. 1957;1957(3):178-82.

6. Echi PC, Ezenwaji HMG. The parasite fauna of characids' (Osteichthyes: Characidae) Anambra River, Nigeria. Afr J Ecol. 2010;48:1-4.

7. Paperna I. Monogenetic Trematodes of the fish of the Volta Basin and South Ghana. Bull Inst Fondam d'Afrique Noire. 1969;31:840-80.

8. Paperna I. Monogenea of inland water fish in Africa. Ann Museé R I'Afrique Cent Sci Zool. 1979;226:1-131.

9. Zaostrovtseva SK. Analysis of fish parasitofauna in Vistula Lagoon (the Baltic Sea). Inland Water Biol. 2009:2:377-82.

10. Zaostrovtseva SK, Evdokimova EB. The ecological analysis of the fish monogenean fauna in Vistula Lagoon (the Baltic Sea), Rivers Pregolja and Prohladnaja. Nat Alm Biol Sci. 2008;10:61-74.

11. Batra V. Prevalence of helminth parasites in three species of cichlids from a man-made lake in Zambia. Zool J Linn Soc. 1984;82:319-33.

12. Yildirim YB, Zeren A, Genc E, Erol C, Konas E. Parasitological investigation on commercially important fish and crustacean species collected from the TIGEM (Dortyol, Turkey) ponds. J Anim Vet Adv. 2010;9:1597-602.

13. Nicoll W. A reference list of the trematode parasites of British freshwater fishes. Parasitology. 1924;16:127-44.

14. Nicoll W. A list of the trematode parasites of British marine fishes. Parasitology. 1915;7:339-78.

15. Lucký Z, Navrátil S, Jirásková M. The health status of commercial fish in the Mušov Lake. Acta Vet Brno. 1989;58:53-90.

16. Mollaret I, Jamieson BGM, Justine JL. Phylogeny of the Monopisthocotylea and Polyopisthocotylea (Platyhelminthes) inferred from 285 rDNA sequences. Int J Parasitol. 2000;30:171-85.

17. Přikrylová I, Mašová Š, Gelnar M, Matla MM, Tavakol S, Luus-Powell WJ. Redescription of the genus Afrodiplozoon Khotenovski, 1981 and its only known species Afrodiplozoon polycotyleus (Paperna, 1973) (Monogenea: Diplozoidae) using a combined multidisciplinary approach. Parasitol Int. 2018:67:245-52.

18. Jovelin $R$, Justine JL. Phylogenetic relationships within the polyopisthocotylean monogeneans (Platyhelminthes) inferred from partial 285 rDNA sequences. Int J Parasitol. 2001;31:393-401. 
19. Littlewood D, Olson PD. Small subunit rDNA and the Platyhelminthes: signal, noise, conflict and compromise. In: Littlewood D, Bray RA, editors. Interrelationships of the Platyhelminthes. 1st ed. London: CRC Press; 2001. p. 262-78.

20. Olson PD, Littlewood DTJ. Phylogenetics of the Monogenea — evidence from a medley of molecules. Int J Parasitol. 2002;32:233-44.

21. Hebert PDN, Cywinska A, Ball SL, DeWaard JR. Biological identifications through DNA barcodes. Proc R Soc London B. 2003;270:313-21.

22. Nishihira T, Urabe M. Morphological and molecular studies of Eudiplozoon nipponicum (Goto, 1891) and Eudiplozoon kamegaii sp. n. (Monogenea; Diplozoidae). Folia Parasitol. 2020;67:018.

23. Jedličková L, Dvoráková H, Kašný M, Ilgová J, Potěšil D, Zdráhal Z, et al. Major acid endopeptidases of the blood-feeding monogenean Eudiplozoon nipponicum (Heteronchoinea: Diplozoidae). Parasitology. 2016;143:494-506.

24. Jedličková L, Dvořáková H, Dvořák J, Kašný M, Ulrychová L, Vorel J, et al. Cysteine peptidases of Eudiplozoon nipponicum: a broad repertoire of structurally assorted cathepsins $L$ in contrast to the scarcity of cathepsins $B$ in an invasive species of haematophagous monogenean of common carp. Parasit Vectors. 2018;11:142.

25. Ilgová J, Jedličková L, Dvořáková H, Benovics M, Mikeš L, Janda L, et al. A novel type I cystatin of parasite origin with atypical legumain-binding domain. Sci Rep. 2017;7:17526.

26. Jedličková L, Dvořák J, Hrachovinová I, Ulrychová L, Kašný M, Mikeš L. A novel Kunitz protein with proposed dual function from Eudiplozoon nipponicum (Monogenea) impairs haemostasis and action of complement in vitro. Int J Parasitol. 2019:49:337-46.

27. Roudnický P, Vorel J, llgová J, Benovics M, Norek A, Jedličková L, et al. Identification and partial characterization of a novel serpin from Eudiplozoon nipponicum (Monogenea, Polyopisthocotylea). Parasite. 2018;25:61.

28. Zhang D, Zou H, Wu SG, Li M, Jakovlić I, Zhang J, et al. Three new Diplozoidae mitogenomes expose unusual compositional biases within the Monogenea class: implications for phylogenetic studies. BMC Evol Biol. 2018;18:133.

29. Sicard M, Desmarais E, Lambert A. Caractérisation moléculaire des populations de Diplozoidae sur cinq espèces de Cyprinidae: nouvelles données sur la spécificité parasitaire. Compt Rend Acad Sci Paris. 2001;324:709-17.

30. Civáňová K, Koyun M, Koubková B. The molecular and morphometrical description of a new diplozoid species from the gills of the Garra rufa (Heckel, 1843) (Cyprinidae) from Turkey- including a commentary on taxonomic division of Diplozoidae. Parasitol Res. 2013;112:3053-62.

31. Fan $L X$, Meng FY, Bai JP, Xu WJ, Wang X. Paradiplozoon yunnanensis $n$. sp. (Monogenea, Diplozoidae) from Sikukia gudgeri (Cyprinidae, Barbinae) in southwest China. Parasite. 2018;25:46.

32. Jirsová D, Ding X, Civáňová K, Jirounková E, llgová J, Koubková B, et al. Redescription of Paradiplozoon hemiculteri (Monogenea, Diplozoidae) from the type host Hemiculter leucisculus, with neotype designation. Parasite. 2018;25:4.

33. Kumar S, Stecher G, Tamura K. MEGA7: Molecular Evolutionary Genetics Analysis version 7.0 for bigger datasets. Mol Biol Evol. 2016;33:1870-4.

34. Kırcalar F, Turgay E, Yardımcı RE, Soylu E, Akmirza A. Molecular identification of Eudiplozoon nipponicum (Gotto, 1891) in Igneada Mert Lake carp (Cyprinus carpio). In: The 19th National Fisheries Symposium. Sinop, Turkey; 2017. p. 206.

35. Ahmad F, Fazili KM, Sofi TA, Sheikh BA, Waza AA, Rashid R, et al. Morphological and molecular characterization of Diplozoon kashmirensis; D. aegyptensis and D. guptai collected from fishes of Kashmir Valley-India. Fish Aquac J. 2015;06:1000147.

36. Ahmad F, Fazili KM, Sofi TA, Waza AA, Rashid R. Comparative molecular characterization of three Diplozoon species from fishes of Kashmir Valley. Agric Adv. 2015;4:65-83.

37. Unal MC, Innal D, Civáňová K, Stavrescu-Bedivan MM, Koubková B, Ozmen $\mathrm{O}$, et al. Identification of two gill parasites Lamproglena compacta (Copepoda: Lernaeidae) and Paradiplozoon bliccae (Monogenea: Diplozoidae) from endemic Aegean chub (Squalius fellowesii). Bull Eur Assoc Fish Pathol. 2017;37:135-47.

38. Vanhove M, Tessens B, Schoelinck C, Jondelius U, Littlewood DTJ, Artois T, et al. Problematic barcoding in flatworms: a case-study on monogeneans and rhabdocoels (Platyhelminthes). ZooKeys. 2013;365:355-79.
39. Pugachev ON, Gerasev PI, Gussev AV, Ergens R, Khotenowsky I. Guide to Monogenea of freshwater fish of Palaeartic and Amur Regions. 1st ed. Milan: Ledizioni-Ledipublishing; 2010.

40. Gao Q, Chen MX, Yao WJ, Gao Y, Song Y, Wang GT, et al. Phylogeny of diplozoids in five genera of the subfamily Diplozoinae Palombi, 1949 as inferred from ITS-2 rDNA sequences. Parasitology. 2007;134:695-703.

41. Avenant-Oldewage A, le Roux LE, Mashego SN, van Vuuren BJ. Paradiplozoon ichthyoxanthon n. sp. (Monogenea: Diplozoidae) from Labeobarbus aeneus (Cyprinidae) in the Vaal River, South Africa. J Helminthol. 2014;88:166-72.

42. Bachellerie JP, Qu LHLH. Ribosomal RNA probes for detection and identification of species. In: Hyde JE, editor. Methods in molecular biology: protocols in molecular parasitology. Totowa: Humana Press; 1993. p. 249-63.

43. Katoh K, Standley DM. MAFFT Multiple Sequence Alignment Software version 7: improvements in performance and usability. Mol Biol Evol. 2013;30:772-80.

44. Altschul SF, Gish W, Miller W, Myers EW, Lipman DJ. Basic local alignment search tool. J Mol Biol. 1990;215:403-10.

45. Bouckaert R, Heled J, Kühnert D, Vaughan T, Wu C-H, Xie D, et al. BEAST 2: a software platform for Bayesian evolutionary analysis. PLoS Comput Biol. 2014;10:e1003537.

46. Nei M, Kumar S. Molecular evolution and phylogenetics. Oxford: Oxford University Press; 2000.

47. Lambert A, Denis A. Étude de l'oncomiracidium de Diplozoon nipponicum Goto, 1981: Hapteur larvaire d'un type nouveau pour le genre Diplozoon (Monogenea, Discocotylidae). Ann Parasitol Hum Comp. 1982;57:533-42.

48. Sicard M, Desmarais E, Vigneux F, Shimazu T, Lambert A. Molecular phylogeny of the Diplozoidae (Monogenea, Polyopisthocotylea) parasitizing 12 species of Cyprinidea (Teleostei): new data about speciation. In: Combes C, Jourdane J, editors. Taxonomy, ecology and evolution of metazoan parasites. Perpignan: PUP; 2003. p. 199-211.

49. Matejusová I, Koubková B, Cunningham CO. Identification of European diplozoids (Monogenea, Diplozoinae) by restriction digestion of the ribosomal RNA internal transcribed spacer. J Parasitol. 2004;90:817-22.

50. Dos Santos QM, van Vuuren BJ, Avenant-Oldewage A. Paradiplozoon vaalense n. sp. (Monogenea: Diplozoidae) from the gills of moggel, Labeo umbratus (Smith, 1841) in the Vaal River System, South Africa. J Helminthol. 2015;89:58-67.

51. Dos Santos QM, Avenant-Oldewage A. The description of a new diplozoid species, Paradiplozoon krugerense n. sp., from Labeo rosae Steindachner, 1894 and Labeo congoro Peters, 1852 in the Kruger National Park, South Africa with notes on the effect of water quality on its infection variables. Hydrobiologia. 2016;777:225-41.

52. Xia X, Xie Z, Salemi M, Chen L, Wang Y. An index of substitution saturation and its application. Mol Phylogenet Evol. 2003;26:1-7.

53. Xia X. DAMBE6: new tools for microbial genomics, phylogenetics, and molecular evolution. J Hered. 2017;108:431-7.

54. Gelnar M, Koubková B, Pláňková H, Jurajda P. Report on metazoan parasites of fishes of the River Morava with remarks on the effects of water pollution. Helminthologia. 1994;31:47-56.

55. Matejusová l, Koubková B, Gelnar M, Cunningham CO. Paradiplozoon homoion Bychowsky \& Nagibina, 1959 versus P. gracile ReichenbachKlinke, 1961 (Monogenea): two species or phenotypic plasticity? Syst Parasitol. 2002;53:39-47.

56. Prost M. Fish Monogenea of Poland. VII. Parasites of Gobio gobio (L.) and Leucaspius delineatus (Heck.). Acta Parasitol Pol. 1984;29:291-7.

57. Le Brun N, Renaud F, Lambert A. The genus Diplozoon (Monogenea, Polyopisthocotylea) in southern France: speciation and specificity. Int J Parasitol. 1988;18:395-400.

58. Shimazu T, Kobayashi K, Tojo K, Besprozvannykh W, Ogawa K. Paradiplozoon skrjabini (Monogenea, Diplozoidae), an ectoparasite on the gills of freshwater fishes (Cyprinidae, Leuciscinae) of Japan and Primorsky Region, Russia: a morphological and molecular study. Bull Natl Museum Nat Sci Ser A. 2015:41:137-54.

59. Dos Santos QM, Avenant-Oldewage A. Soft tissue digestion of Paradiplozoon vaalense for SEM of sclerites and simultaneous molecular analysis. J Parasitol. 2015;101:94-7.

60. Dos Santos QM, Dzika E, Avenant-Oldewage A. Using scanning electron microscopy (SEM) to study morphology and morphometry of the isolated haptoral sclerites of three distinct diplozoid species. PLOS ONE. 2019;14:e21179. 
61. Pandey KC, Agrawal N. An encyclopaedia of Indian Monogenoidea. 1st ed. New Delhi: Vitasta Publishing; 2008.

62. Froese R, Pauly D. FishBase. https://www.fishbase.org. Accessed 6 Sep 2020.

63. Wu B, Lang S, Wang W. Fauna Sinica: Platyhelminthes: Monogenea. Beijing: Science Press; 2000.

64. Chakrabarty P. Genetypes: a concept to help integrate molecular phylogenetics and taxonomy. Zootaxa. 2010;2632:67-8.

65. Pleijel E, Jondelius U, Norlinder E, Nygren A, Oxelman B, Schander C, Sundberg $\mathrm{P}$, Thollesson M. Phylogenies without roots? A plea for the use of vouchers in molecular phylogenetic studies. Mol Phylogenet and Evol. 2008;48:369-71.

\section{Publisher's Note}

Springer Nature remains neutral with regard to jurisdictional claims in published maps and institutional affiliations.
Ready to submit your research? Choose BMC and benefit from:

- fast, convenient online submission

- thorough peer review by experienced researchers in your field

- rapid publication on acceptance

- support for research data, including large and complex data types

- gold Open Access which fosters wider collaboration and increased citations

- maximum visibility for your research: over $100 \mathrm{M}$ website views per year

At BMC, research is always in progress.

Learn more biomedcentral.com/submissions 